\title{
Determination of the barycentric velocity of an astrometric satellite using its own observational data
}

\author{
A. G. Butkevich ${ }^{1} \dagger$ and S. A. Klioner ${ }^{2}$ \\ Lohrmann Observatory, Dresden Technical University, \\ 01062 Dresden, Germany \\ ${ }^{1}$ email: alexey.butkevich@tu-dresden.de \\ ${ }^{2}$ email: sergei.klioner@tu-dresden.de
}

\begin{abstract}
The problem of determination of the orbital velocity of an astrometric satellite from its own observational data is studied. It is well known that data processing of microarcsecondlevel astrometric observations imposes very stringent requirements on the accuracy of the orbital velocity of satellite (a velocity correction of $1.45 \mathrm{~mm} / \mathrm{s}$ implies an aberrational correction of 1 $\mu$ as). Because of a number of degeneracies the orbital velocity cannot be fully restored from observations provided by the satellite. Seven constraints that must be applied on a velocity parameterization are discussed and formulated mathematically. It is shown what part of velocity can be recovered from astrometric data using a combined fit of both velocity parameters and astrometric parameters of the sources. Numerical simulations show that, with the seven constraints applied, the velocity and astrometric parameters can be reliably estimated from observational data. It is also argued that the idea to improve the velocity of an astrometric satellite from its own observational data is only useful if a priori information on orbital velocity justifies the applicability of the velocity constraints. The proposed model takes into account only translational motion of the satellite and ignores any satellite-specific parameters. Therefore, the results of this study are equally applicable to both scanning missions similar to Gaia, and pointing ones like SIM, provided that enough sources were observed sufficiently uniformly.
\end{abstract}

Keywords. astrometry, methods: data analysis, ephemerides, reference systems

\section{Introduction}

The astrometric accuracy of several microarcseconds announced for three space missions (Gaia, SIM and Jasmine) implies that the velocity of the satellite should be known within several $\mathrm{mm} / \mathrm{s}$. Such a high precision is a challenge to standard orbit determination techniques.

Another way to proceed was discussed in the Gaia community since 2001 and formulated in the written form by Klioner (2005). The idea is to use Gaia's own astrometric data to fit a correction to the Gaia velocity. The velocity correction $\delta \mathbf{v}$ is the difference between the real velocity of the satellite and its ephemeris velocity available a priori. A straightforward theoretical analysis of this idea (Butkevich 2006) demonstrated that velocity can be determined from observations with the required precision, provided that all other parameters are exactly known. Some criticism against this approach has been formulated by Bastian (2004a) who argued that the velocity obtained from observations will strongly correlate with the source parameters. A detailed theoretical study of this problem has been done by Klioner \& Butkevich (2007), who explicitly demonstrated that the velocity correction to be fitted from the data must satisfy some constraints.

$\dagger$ On leave from Pulkovo Observatory, 196140 Saint-Petersburg, Russia. 


\section{What part of observer's velocity can be restored from astrometric data?}

Let us consider the case when the velocity correction has a constant component:

$$
\delta \mathbf{v}=\mathbf{v}_{0}=\text { const },
$$

then this additional velocity leads to the following aberrational correction

$$
\delta \mathbf{u} \approx-\mathbf{u} \times\left[\mathbf{u} \times \mathbf{v}_{0} / c\right] .
$$

This correction depends on the stellar position $\mathbf{u}$, but for each star it is constant. Such a signal in $\delta \mathbf{v}$ is equivalent to a constant change of positions, which cannot be detected from observations since it cannot be distinguished from their real change.

Similarly, for a velocity correction changing linearly with time,

$$
\delta \mathbf{v}=\mathbf{a}_{0} t \quad \mathbf{a}_{0}=\text { const },
$$

we have

$$
\delta \mathbf{u}(t) \approx \boldsymbol{\mu}_{0} t
$$

where

$$
\boldsymbol{\mu}_{0}=-\mathbf{u} \times\left[\mathbf{u} \times \mathbf{a}_{0} / c\right] .
$$

This correction is equivalent to an additional constant proper motion for each star. Such a correction again cannot be detected from observations since it cannot be distinguished from a real change of the proper motion parameters for each source.

If $\delta \mathbf{v}$ is exactly proportional to the barycentric position of the satellite $\mathbf{r}(t)$ :

$$
\delta \mathbf{v}(t)=\alpha_{0} \mathbf{r}(t) \quad \alpha_{0}=\text { const },
$$

the corresponding first-order aberrational correction reads

$$
\delta \mathbf{u} \approx-\mathbf{u} \times\left[\mathbf{u} \times \alpha_{0} \mathbf{r} / c\right] .
$$

On the other hand, the aberrational effect caused by a global offset of parallaxes $\delta \pi$ is

$$
\delta \mathbf{u}=\mathbf{u} \times[\mathbf{u} \times \delta \pi \mathbf{r} / \mathrm{AU}] .
$$

These effects are indistinguishable provided that

$$
\delta \pi=-\alpha_{0} \mathrm{AU} / c .
$$

Thus the problem has seven free parameters $\left(\alpha_{0}\right.$ and six components of $\mathbf{v}_{0}$ and $\left.\mathbf{a}_{0}\right)$, which correlate with some astrometric information, i. e. it has seven degrees of freedom. This rank deficiency makes the direct determination of velocity impossible. It can be, however, demonstrated that the degeneracy can be eliminated if the following constraints would be imposed onto the solution:

$$
\begin{array}{rlr}
\int_{0}^{T} \delta \mathbf{v}(t) \mathrm{d} t=0 & \text { to remove } & \delta \mathbf{v}=\mathbf{v}_{0}, \\
\int_{0}^{T}(t-T / 2) \delta \mathbf{v}(t) \mathrm{d} t & =0 \quad \text { to remove } & \delta \mathbf{v}=\mathbf{a}_{0} t, \\
\int_{0}^{T} \frac{\delta \mathbf{v}(t) \mathbf{r}(t)}{|\mathbf{r}(t)|^{2}} \mathrm{~d} t=0 & \text { to remove } & \delta \mathbf{v}=\alpha_{0} \mathbf{r} .
\end{array}
$$


Table 1. Singular values of the normal matrix.

\begin{tabular}{|c|c|c|c|c|}
\hline$n$ & 1 & 7 & 8 & $\max$ \\
\hline$\sigma_{n}$ & $9.6 \cdot 10^{-8}$ & $9.0 \cdot 10^{-6}$ & 2.1 & 1045 \\
\hline
\end{tabular}

Table 2. Error in parameters.

\begin{tabular}{ccccccccc}
\hline Parameter & $\alpha$ & $\delta$ & $\mu_{\alpha}$ & $\mu_{\delta}$ & $\pi$ & $\delta v_{x}$ & $\delta v_{y}$ & $\delta v_{z}$ \\
\hline Error & $1.6 \mu$ as & $1.5 \mu$ as & $1.3 \mu$ as $/$ yr & $1.2 \mu$ as $/$ yr & $1.5 \mu$ as & $1.8 \mathrm{~mm} / \mathrm{s}$ & $1.5 \mathrm{~mm} / \mathrm{s}$ & $1.6 \mathrm{~mm} / \mathrm{s}$ \\
\hline
\end{tabular}

These constraints guarantee that the solution does not contain the relevant signals in the sense of least-squares.

Although it can hardly be proven analytically that the problem has no other degrees of freedom, it can be checked numerically. We calculated singular value decomposition (SVD) of a normal matrix and found only seven small singular values shown in Table 1. This indicates that no other degree of freedom exists

\section{Legitimacy of the constrained velocity}

The constraints may only be applied when a priori accuracy of the ephemeris guarantees that no signal of a given kind can exist in real velocity, or, strictly speaking, it is so small that any effect due to it can be neglected.

The real $\delta \mathbf{v}_{\text {real }}$ velocity correction can be represented as a sum of the two different components

$$
\delta \mathbf{v}_{\text {real }}=\delta \mathbf{v}+\mathbf{R}(t),
$$

where $\delta \mathbf{v}$ is the component that can be fitted and $\mathbf{R}(t)$ is the component violating the seven constraints. The fitted component $\delta \mathbf{v}$ is useful if and only if the uncertainty of the ephemeris velocity is such that it guarantees that

$$
|\mathbf{R}(t)|<\epsilon
$$

at any instant of time. Here $\epsilon$ is a required velocity accuracy (for Gaia, $\epsilon=1 \mathrm{~mm} / \mathrm{s}$ ).

Fortunately, this can be demonstrated for Gaia (Klioner \& Butkevich, 2007) but cannot be guaranteed for other missions.

\section{Results of numerical simulations}

To study the problem of the velocity determination numerically, we have implemented a simple simulator of Gaia observations - Dresden Gaia Simulator (DGS) - that includes many basic features of a real mission. Table 2 shows the results of a simulation run with 2048 stars covering 5 years of observations (Butkevich \& Klioner 2007a). The accuracy of an individual observation was chosen to be $30 \mu$ as. The small errors found suggest that the constrained solution allows one to achieve a precise and reliable determination of velocity and source parameters.

\section{Assessment of the accuracy of velocity determination}

Our simulation has one serious drawback - it can only handle limited datasets and never approaches the data volume near to the expected Gaia output. A straightforward 


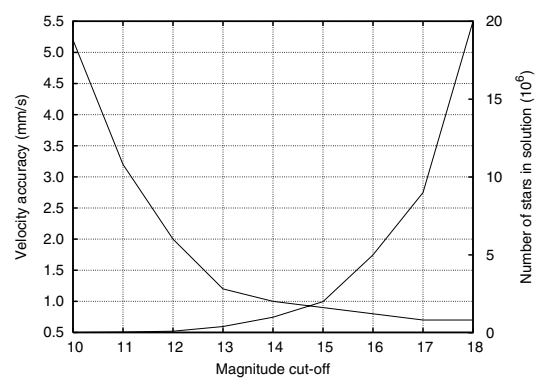

Figure 1. Dependence of the velocity accuracy (the descending curve) and the number of stars in the astrometric solution (the ascending curve) on the limiting magnitude.

estimate can be obtained using simple statistical considerations (Butkevich \& Klioner $2007 \mathrm{~b})$. One of the critical parameters in the velocity determination is the number of stars used in the solution, which depends on the apparent magnitude cut-off. The estimated velocity accuracy together with relevant star counts are shown in Fig. 1 for the limiting $V$ magnitudes. Besides the number of stars, the accuracy also depends on the temporal resolution of the fitted velocity correction. The accuracy obviously degrades when a finer resolution is used. The time scale of velocity variations was chosen to be 6 hours, close to the rotation period of Gaia. From our estimates, we conclude that at least $10^{6}$ stars are needed to obtain velocity with an accuracy of $1 \mathrm{~mm} / \mathrm{s}$.

\section{Satellite specific parameters}

The discussed model takes into account only translational motion of the satellite and ignores any satellite-specific calibration parameters (e. g. attitude parameters). The situation may become more complicated when those other parameters are also considered. Our analysis shows that for Gaia the scientifically important parameters can be successfully restored even in this case.

\section{Acknowledgements}

We acknowledge useful discussions with Ulrich Bastian and Lennart Lindegren. This work was partially supported by the BMWi grant 50 QG 0601 awarded by the Deutsche Zentrum für Luft- und Raumfahrt e.V. (DLR).

\section{References}

Bastian, U. 2004a, Improving Gaia's orbit with Gaia's astrometry?, GAIA-ARI-BAS-007 Butkevich, A. G. 2006, On the velocity determination from observational data, GAIA-CA-TNLO-AGB-001-1

Butkevich, A. G. \& Klioner, S. A. 2007a, On the simultaneous determination of velocity correction and source parameters, GAIA-CA-TN-LO-AGB-002-1

Butkevich, A. G. \& Klioner, S. A. 2007b, Assessing the accuracy of the velocity determination, GAIA-CA-TN-LO-AGB-005-1

Klioner, S. 2005, On the possibility to improve the velocity of Gaia from the Gaia's own astrometric data, available from Gaia Livelink

Klioner, S. A. \& Butkevich, A. G. 2007, What part of observer's velocity can be restored from astrometric data?, GAIA-CA-TN-LO-SK-001-2 